Ann. Sci. forest., 1979, 36 (3), 231-237.

\title{
Caractères morphologiques et teneurs en éléments minéraux des aiguilles portées sur les différentes pousses (de printemps et «d'août») chez l'Epicéa commun (Picea excelsa Link)
}

\author{
G. LEVY et D. G. STRULLU * \\ avec la collaboration technique de Y. LEFEVRE \\ Station de Recherches sur les Sols forestiers et la Fertilisation \\ Centre National de Recherches forestières, I.N.R.A. \\ Champenoux, 54280 Seichamps \\ * Laboratoire de Botanique L. Daniel, \\ U.E.R. des Sciences Biologiques \\ 35042 Rennes Cedex
}

\section{Résumé}

Cette étude montre que, chez l'Epicéa, la longueur, la largeur et l'épaisseur des feuilles varient en fonction de leur position sur le jeune plant : ainsi, les feuilles situées sur les pousses d'août sont plus larges et plus épaisses que celles formées au printemps.

A ces différences morphologiques correspondent des variations dans les teneurs en éléments minéraux. En particulier les feuilles portées par les pousses d'août présentent les teneurs les plus élevées en phosphore et en potassium et les teneurs les plus basses en azote et en calcium.

\section{I. - Introduction}

Le diagnostic foliaire est une technique couramment utilisée en matière forestière ; en particulier, on compare la teneur des feuilles en éléments nutritifs aux valeurs de l'optimum et du seuil de carence. Ainsi, chez de jeunes plants d'Epicéa commun, on analyse habituellement les aiguilles portées par les pousses du dernier verticille. Or les plants présentent une morphologie variable résultant des caractéristiques du développement. Fréquemment, les premières pousses de l'année, développées au printemps, se prolongent après un arrêt de la croissance par des « pousses d'août ». La fréquence de ce phénomène dépend de facteurs génétiques (provenances) et de facteurs du milieu (conditions climatiques et nutritives en particulier). A la suite de l'examen des caractères morphologiques des feuilles portées par les «pousses d'août ", nous avons été amenés à nous demander si à la variabilité des caractères foliaires ne correspond pas une hétérogénéité dans les teneurs en éléments minéraux, ce qui poserait le problème de la constitution de l'échantillon à analyser. Cette étude pourrait alors, outre les précisions qu'elle apporte sur les différences morphologiques entre types d'aiguilles, contribuer à améliorer les diagnostics foliaires utilisés dans certaines expériences en pépinière. 


\section{II. - Matériel et méthodes}

Les jeunes plants de provenance Bonnétage (Jura) ont été prélevés en fin de $2^{\mathrm{e}}$ année de repiquage (plants $2+2$ ). Ils appartiennent aux compartiments témoins de 3 blocs d'un dispositif expérimental et ont reçu une fertilisation minérale compensant les exportations, soit par are : 4,4 kg de superphosphate simple (16 p. 100 de $\left.\mathrm{P}_{2} \mathrm{O}_{5}\right)$, $2,3 \mathrm{~kg}$ de sulfate de potassium ( $48 \mathrm{p} .100$ de $\mathrm{K}_{2} \mathrm{O}$ ) et $1,2 \mathrm{~kg}$ de sulfate de magnésium (16 p. $100 \mathrm{de} \mathrm{MgO}$ ) en engrais de fond mélangés au sol. De plus, les plants ont reçu $7 \mathrm{~kg} /$ are d'ammonitrate à 34 p. 100 d'azote dont le tiers la première année et le complément la seconde année du repiquage en deux doses annuelles, début juin et mi-juillet la première année, quinze jours plus tôt la seconde année, dates qui nous ont fourni par ailleurs les résultats les plus satisfaisants (plants les plus développés).

Les analyses minérales ( $\mathrm{N}, \mathrm{P}, \mathrm{K}, \mathrm{Ca}$ et $\mathrm{Mg}$ ) ont été effectuées sur des aiguilles provenant des diverses pousses en séparant chaque répétition. Les caractères morphologiques foliaires ont été obtenus à partir de mesures effectuées sur coupes transversales pratiquées au milieu des feuilles.

\section{III. - Résultats}

\section{1. - Caractères morphologiques}

3.11. Types de pousses.

Environ 50 p. 100 des plants ayant reçu la fertilisation indiquée ci-dessus n'ont pas de pousses d'août et correspondent au type A (fig. 1). De l'ordre de 30 p. 100 des plants
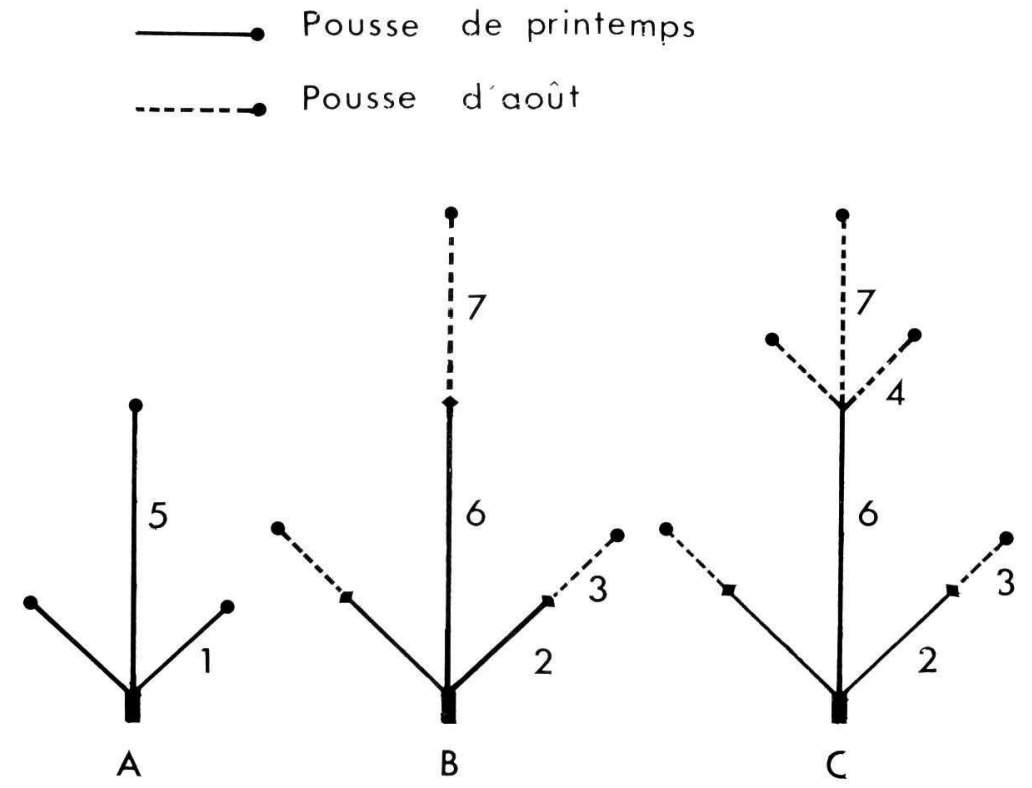

FIG. 1. - Différents types de plants.

FIG. 1. - Different types of plant (with spring and lammas shoots). 
(appartenant au type B) ont développé une " pousse d'août » en position terminale et sur le dernier verticille. Enfin, se rattachent au type $C 20$ p. 100 des plants qui ont en plus développé un nouveau verticille à l'apex de la première pousse de printemps.

La figure 1 schématise la morphologie des plants de type $A$, B et $C$ et montre la variabilité de composition des pousses annuelles terminales et latérales. Cette hétérogénéité est encore accrue en raison du fait que la proportion de chaque type de pousse est également variable, d'un traitement à l'autre, et d'une année sur l'autre pour un même traitement.

3.12. Caractères morphologiques des feuilles.

Les valeurs du $F$ théorique sont de 3,00 à 5 p. 100 et 4,82 à 1 p. 100.

a) Longueur $(\mathrm{mm})\left({ }^{1}\right)$

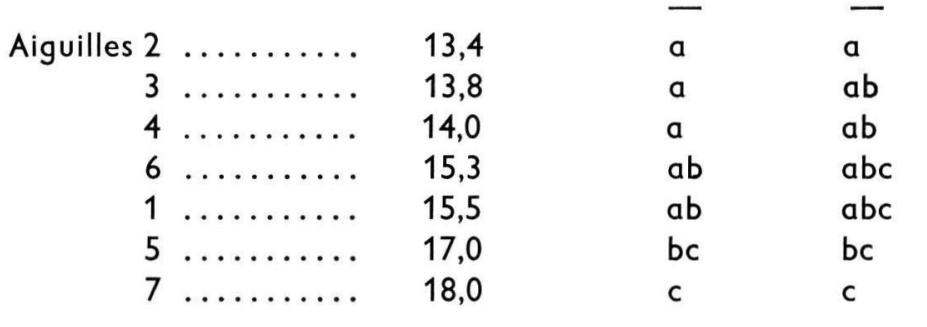

$\mathrm{F}$ calculé $\ldots \ldots \ldots \ldots . \quad 5,43^{++}$

b) Largeur (mm) ( $\left.{ }^{1}\right)$

\begin{tabular}{|c|c|c|c|}
\hline Aiguilles 2 & $\ldots \ldots \ldots \ldots$ & 3,13 & $a$ \\
\hline 1 & 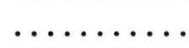 & 3,39 & a \\
\hline 6 & $\ldots \ldots \ldots \ldots$ & 3,70 & $a$ \\
\hline 5 & $\ldots \ldots+\cdots$ & 3,70 & $a$ \\
\hline 4 & 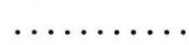 & 4,97 & b \\
\hline 3 & $\ldots \ldots \ldots \ldots$ & 5,08 & b \\
\hline 7 & 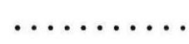 & 6,26 & c \\
\hline
\end{tabular}

$\mathrm{F}$ calculé $\ldots \ldots \ldots \ldots . \quad 34,1^{++}$

c) Epaisseur $(\mathrm{mm})\left({ }^{1}\right)$

\begin{tabular}{|c|c|c|c|c|}
\hline Aiguilles 1 & $\ldots \ldots \ldots \ldots$ & 4,42 & $a$ & $a$ \\
\hline 6 & $\ldots \ldots \ldots$ & 4,48 & $a b$ & a \\
\hline 5 & $\ldots \ldots \ldots \ldots$ & 4,49 & $a b$ & a \\
\hline 2 & $\ldots \ldots \ldots \ldots$ & 4,94 & $a b$ & $a$ \\
\hline 3 & $\ldots \ldots \ldots \ldots$ & 5,03 & $a b$ & $a b$ \\
\hline 4 & $\ldots \ldots \ldots$ & 5,11 & b & $a b$ \\
\hline 7 & $\ldots \ldots \ldots \ldots$ & 5,92 & c & b \\
\hline
\end{tabular}

(1) Les valeurs qui n'ont pas au moins une lettre (a, b ou c) commune sont statistiquement différentes. 
Les feuilles portées par les pousses d'août en position terminale (fig. $1: 7$ ) arrivent en tête pour les trois caractères étudiés. Elles sont significativement plus longues, larges et épaisses, à 5 p. 100, que presque tous les autres types de feuilles ; seule exception, et pour la longueur seulement, les feuilles des pousses de printemps en position terminale non suivies d'une pousse d'août (5).

Par ailleurs ces dernières sont (à 5 p. 100) plus longues que celles portées par les pousses latérales des plants à pousses d'août (2, 3 et 4). Enfin, les feuilles des pousses d'aoûf latérales (3 et 4) sont significativement plus larges que les feuilles portées par tous les autres types de pousses (sauf 7 , bien sûr) ; une tendance identique, mais nettement moins marquée et en général non significative, se manifeste pour l'épaisseur des feuilles.

\section{2. - Composition minérale des aiguilles}

Les aiguilles des pousses terminales n'ont pas été analysées car elles ne le sont pas dans la pratique.

Les valeurs du $F$ théorique sont de 4,76 à 5 p. 100 et de 9,78 à 1 p. 100.

3.21. Azote.

\begin{tabular}{|c|c|c|c|c|}
\hline & & (N p. 100$)$ & 5 p. 100 & 1 p. 100 \\
\hline & & - & - & - \\
\hline Aiguilles 4 & $\ldots \ldots \ldots$ & 2,22 & $a$ & $a$ \\
\hline 3 & $\ldots \ldots \ldots$ & 2,34 & $a b$ & $a b$ \\
\hline 2 & $\ldots \ldots \ldots$ & 2,42 & $b$ & $a b$ \\
\hline 1 & $\ldots \ldots \ldots \ldots$ & 2,63 & c & $b$ \\
\hline F calculé & & $9,33^{+}$ & & \\
\hline
\end{tabular}

On note surtout que les aiguilles des pousses 1 ont une teneur en azote significativement supérieure à toutes les autres (au seuil 5 p. 100).

3.22. Phosphore et potassium.

\begin{tabular}{|c|c|c|c|c|}
\hline & & $P(p .100)$ & 5 p. 100 & 1 p. 100 \\
\hline & & - & - & - \\
\hline Aiguilles 2 & $\ldots \ldots \ldots \ldots$ & 0,24 & $a$ & $a$ \\
\hline 1 & $\ldots \ldots \ldots$ & 0,25 & $a$ & $a b$ \\
\hline 4 & $\ldots \ldots \ldots$ & 0,28 & $b$ & bc \\
\hline 3 & $\ldots \ldots \ldots \ldots$ & 0,28 & $b$ & c \\
\hline \multirow{3}{*}{\multicolumn{2}{|c|}{ F calculé $\ldots . \ldots \ldots \ldots$}} & $14,30^{++}$ & & \\
\hline & & $K(p .100)$ & 5 p. 100 & 1 p. 100 \\
\hline & & - & - & - \\
\hline Aiguilles 1 & $\ldots \ldots \ldots \ldots$ & 0,50 & $a$ & $a$ \\
\hline 2 & $\ldots \ldots \ldots$ & 0,54 & $a$ & $a$ \\
\hline 3 & $\ldots \ldots \ldots$ & 0,71 & $b$ & $b$ \\
\hline 4 & $\ldots \ldots \ldots \ldots$ & 0,76 & $b$ & $b$ \\
\hline F calculé & $\ldots \ldots \ldots$ & $88,81^{++}$ & & \\
\hline
\end{tabular}


Phosphore et potassium offrent des résultats assez comparables et très différents de ceux qui concernaient l'azote : quels que soient les types de plants dont proviennent les pousses, les aiguilles des pousses d'août sont plus riches que celles des pousses initiales.

3.23. Calcium.

\begin{tabular}{|c|c|c|c|c|}
\hline & & $\mathrm{Ca}(p .100)$ & 5 p. 100 & 1 p. 100 \\
\hline & & - & - & - \\
\hline Aiguilles 4 & $\ldots \ldots \ldots \ldots$ & 1,35 & $a$ & $a$ \\
\hline 3 & $\ldots \ldots \ldots \ldots$ & 1,70 & $b$ & $a b$ \\
\hline 2 & $\ldots \ldots \ldots \ldots$ & 1,85 & bc & $b$ \\
\hline 1 & $\ldots \ldots \ldots$ & 2,05 & c & $b$ \\
\hline F calculé & $\ldots \ldots \ldots \cdots$ & $15,31++$ & & \\
\hline
\end{tabular}

Les résultats sont assez voisins de ceux obtenus pour l'azote.

3.24. Magnésium.

\begin{tabular}{|c|c|c|c|c|}
\hline & & $\mathrm{Mg}($ p. 100) & 5 p. 100 & 1 p. 10() \\
\hline & & - & - & - \\
\hline Aiguilles 4 & $\ldots \ldots \ldots \ldots$ & 0,050 & $a$ & $a$ \\
\hline 2 & $\ldots \ldots \ldots$ & 0,056 & $a$ & $a$ \\
\hline 3 & $\ldots \ldots \ldots$ & 0,076 & $b$ & $b$ \\
\hline 1 & $\ldots \ldots \ldots \ldots$ & 0,076 & $b$ & $b$ \\
\hline F calculé & $\cdots$ & $22,66++$ & & \\
\hline
\end{tabular}

Ces différences ne peuvent être assimilées à aucun des types précédents.

\section{IV. - Discussion et conclusion}

Cette étude montre en particulier que, d'un point de vue morphologique, les feuilles formées sur des pousses d'août sont plus larges et ont tendance à être plus épaisses que celles formées au printemps lors de la mise en place de la première pousse. La variabilité des caractères morphologiques foliaires peut être attribuée à divers facteurs dont les conditions climatiques (Aussenac, 1973) et les conditions de nutrition (Leroy, Levy, Strullu, 1973). Dans le cas présent, on peut penser que les différences climatiques entre le printemps ef l'été ont joué un rôle primordial. Les possilités de nutrition (sels minéraux et eau) à ces deux périodes de l'année ont également pu varier et avoir un effet.

Les variations notées doivent se traduire par une augmentation du volume foliaire. Les phénomènes de dilution ne peuvent cependant expliquer toutes les données concernant les teneurs foliaires. Les éléments peuvent se classer en 2 groupes :

1) L'azote et le calcium ont des teneurs maximales chez les plants sans pousse d'aoûtou les pousses de printemps des plants à pousse d'août. Bien que les résultats convergent pour les deux éléments, ils ne semblent pas expliquables par les mêmes causes. On sait que les teneurs en calcium augmentent dans les aiguilles en fonction de l'âge ; 
les feuilles des pousses de printemps, plus âgées, sont plus riches que les feuilles des "pousses d'août ». L'azote migre facilement dans le sol ; il est possible que lors de la formation des pousses d'août les plants avaient moins d'azote à leur disposition. Le fait que les aiguilles des plants sans pousse d'août soient les plus riches de toutes en azote peut faire penser aussi à un phénomène de dilution dans les autres types de plants (migration de l'azote des aiguilles de printemps vers celles des pousses d'août).

2) Le phosphore et le potassium offrent des teneurs maximales dans les feuilles portées par les « pousses d'août ». Pour expliquer ces différences on pourrait avancer l'argument inverse de celui énoncé pour le calcium : les concentrations foliaires en $P$ et $\mathrm{K}$ diminuent en général avec l'âge des aiguilles.

Quant aux résultats qui concernent le magnésium, aucune explication ne peut être avancée pour l'instant.

Nos résultats sont souvent très proches de ceux cités par différents auteurs bien qu'il s'agisse généralement de phénomènes morphologiques difficilement comparables à la pousse d'août de l'Epicéa :

\begin{tabular}{|c|c|c|c|c|}
\hline & $N(p .100)$ & $P($ p. 100) & K (p. 100) & $\mathrm{Ca}(\mathrm{p} .100)$ \\
\hline \multicolumn{5}{|l|}{$\begin{array}{l}\text { - Czerney ef Fiedler (1968) } \\
\text { - Mélèze en août: }\end{array}$} \\
\hline feuilles de pousses courtes (printemps) & 2,0 & 0,08 & 0,50 & 0,42 \\
\hline $\begin{array}{l}\text { feuilles de pousses longues (été) } \ldots \text {. } \\
\text { - Chêne en août : }\end{array}$ & 1,5 & 0,08 & 0,92 & 0,30 \\
\hline fevilles de printemps............... & 1,7 & 0,08 & 0,90 & 0,61 \\
\hline $\begin{array}{l}\text { feuilles de " pousses de St-Jean " } \ldots \\
\text { — Hohne (1976) sur Mélèze }\end{array}$ & 2,0 & 0,14 & 1,10 & 0,64 \\
\hline feuilles de pousses courtes (printemps) & 2,0 & 0,22 & 0,77 & 0,75 \\
\hline $\begin{array}{l}\text { feuilles de pousses longues (été) } \\
\text { - Bonneau (non publié) sur chêne vert } \\
\text { de } 2 \text { ans fertilisé en NPKCa, analyse } \\
\text { en novembre }\end{array}$ & 1,8 & 0,31 & 1,16 & 0,67 \\
\hline fevilles de printemps.............. & 1,48 & 0,12 & 0,76 & 0,51 \\
\hline fevilles d'été $\ldots \ldots \ldots \ldots \ldots \ldots \ldots$ & 1,58 & 0,17 & 1,02 & 0,21 \\
\hline
\end{tabular}

En conclusion, cette étude apporte la preuve que pour effectuer le diagnostic foliaire, on ne peut prélever des aiguilles indifféremment sur les diverses pousses des jeunes plants en pépinière. On pourrait être tenté de recueillir pour chacune d'elles un nombre correspondant à sa fréquence et effectuer des analyses sur l'ensemble des aiguilles provenant d'un mélange ; c'est d'ailleurs ce qui est pratiqué de façon habituelle en dehors du fait que les proportions sont prises au hasard. II nous paraît plus judicieux de comparer des lots homogènes.

De plus, cette étude a montré que les pousses d'août donnaient des résultats très variables, ce qui peut conduire à les éliminer et à ne retenir que les pousses latérales de printemps (1 et 2) provenant indifféremment des plants aoûtés ou non ( $A, B, C)$. Cependant, en fonction des données obtenues pour l'azote (cf. 3.21), il paraît préférable d'analyser séparément les pousses de printemps issues de plants sans et avec pousses d'août (A d'une part, B-C d'autre part). On pourrait même plus simplement ne constituer que des échantillons issus de plants monocycliques (A).

$\mathrm{Si}$ l'on retient cette dernière solution (uniquement pousses 1 ), divers cas peuvent se présenter :

1) Si l'on désire avoir une idée du niveau général de nutrition de plants issus d'une 
pépinière de production par exemple, et dont un certain pourcentage possède des pousses d'août, il sera nécessaire de tenir compte lors de l'interprétation des résultats, du fait que les plants choisis pour l'échantillonnage ne sont sans doute pas les plus vigoureux de la population.

2) Dans une expérience en pépinière, afin de comparer la nutrition des divers traitements, l'échantillon prélevé dans les traitements où les plants à pousse d'août sont très nombreux sera forcément inférieur à la moyenne. Alors, afin de comparer dans toute la mesure du possible les mêmes tranches de population, on sera amené à choisir également, dans les traitements où il n'y a pas ou peu de plants à pousses d'août, des plants de taille inférieure à la moyenne du traitement.

3) Cas des plantations (plants plus âgés). II serait intéressant de vérifier si l'on obtient des résultats semblables à ceux de pépinière ; dans l'affirmative, il faudrait en tirer les mêmes conséquences pour l'échantillonnage en vue du diagnostic foliaire.

Mais l'objectif de l'analyse pourra être autre que le diagnostic foliaire ; il conditionnera alors le choix de l'échantillon. Ainsi, l'estimation des « exportations » d'éléments nutritifs en pépinière nécessite l'analyse de l'ensemble des feuilles d'un certain nombre de plants représentatifs.

Reçu pour publication en novembre 1978.

\section{Summary}

Morphological characteristics and mineral nutrients content of the needles growing on the different shoots (spring and lammas shoots) of Norway Spruce (Picea excelsa Link)

This study shows that, on Norway Spruce, the length, width and thickness of the needles vary according to their position on the young plant : thus, the needles growing on lammas shoots are wider and thicker than the ones that grow during the spring. There are also some variations in the mineral nutrients content. In particular, the needles growing on lammas shoots have a greater content in phosphorus and potassium and a smaller one in nitrogen and calcium. It is necessary to take these differences into account when sampling with a view to foliar diagnosis.

\section{Références bibliographiques}

AUSSENAC G., 1973. Effets de conditions microclimatiques différentes sur la morphologie et la structure anatomique des aiguilles de quelques résineux. Ann. Sci. forest., 30 (4), 375-392.

CZERNEY P., FIEDLER H. J., 1968. The dynamics of mineral nutrients in the assimilation organs of tree species on fertilized and non fertilized pseudogley. Arch. Forsfw., 17 (12), 1263-1278.

HOHNE H., 1976. Blattanalytische Untersuchungen an jüngeren Larchenständen. Beitrage $f$. der Forstw. (3), 138-147.

LEROY Ph., LEVY G., STRULLU D. G., 1973. Action de la matière organique et des engrais minéraux en pépinière sur la croissance et les caractères foliaires du Douglas. Ann. Sci. Forest., 30 (4), 393-412. 